\title{
Simplified Quantitative Analysis of Breast Thermograms for Breast Asymmetry Estimation
}

\author{
Valery Chernov ${ }^{1}$, Grigory Chernov ${ }^{2}$, Federico Cirett-Galán ${ }^{3}$, Raquel Torres-Peralta ${ }^{3}$ José $^{2}$ \\ Luis Ruiz-Duarte ${ }^{3}$, Enrique Martín-del-Campo-Mena ${ }^{4}$ and Marcelino Barboza-Flores ${ }^{1}$ \\ ${ }^{1}$ Departamento de Investigación en Física, Universidad de Sonora, Hermosillo, Sonora \\ 83000, Mexico \\ ${ }^{2}$ Departamento de Física, Doctorado en Nanotecnología, Universidad de Sonora, Hermosillo, \\ Sonora 83000, Mexico \\ ${ }^{3}$ Departamento de Ingeniería Industrial, Universidad de Sonora, Hermosillo, Sonora, Mexico \\ ${ }^{4}$ Centro Estatal de Cancerología: Miguel Dorantes Mesa, Aguascalientes 100, Progreso \\ Macuiltepetl, Xalapa, Veracruz, 91130, Mexico \\ chernov@cifus.uson.mx
}

Infrared thermography is a unique technology that was approved by the FDA as an adjunct diagnostic breast screening procedure. It can detect early physiologic changes that can be signs of danger in the body years before other tools and offers the opportunity for much earlier detection of cancer. The motivation of the present work is to develop a simple quantitative procedure for estimation of an asymmetry level (mild, moderate or severe) of breast thermograms. These asymmetry levels are not related directly with a possibility of existence of cancer but should help to distribute testing persons into three groups. The first group, with mild asymmetry, will be supposed to have no thermographic abnormalities. Persons from the second group, moderate asymmetry, will be asked to pass a second thermography exam within 3 to 6 months. The third group, persons with severe asymmetry, will be recommended to contact with an oncology doctor and pass the second thermography exam within 2 to 3 months.

100 frontal infrared breast thermograms for patients with breast cancer and 200 thermograms for nominally healthy volunteers were recorded, processed and analyzed in the present study. First, the line of symmetry and regions of interest (ROI, 5 on the right and 5 on left) were manually selected. The line of symmetry was defined as a perpendicular that crosses the line connecting nipple centers in the middle. The ROIs selected are the following body areas: nipple with areola, breast without areola, a part of the chest above the breast, axilla and submammary. Subsequently, hot and cool zones on the right and left breast ROIs, as well as the corresponding contralateral zones on the opposite breast were found. The mean temperatures for all selected ROIs and zones were calculated. To estimate partial asymmetry levels for each ROI and zone the differences between the mean temperatures of right and left body sides were found and compared with predetermined critical values. The total image asymmetry level was defined based on the partial asymmetry levels.

Keywords: infrared thermography, asymmetry, breast cancer. 